\title{
BM-ca is a newly defined type I/II anti-CD20 monoclonal antibody with unique biological properties
}

\author{
MICHIO NISHIDA ${ }^{1}$, NORIO UEMATSU ${ }^{2}$, HIDEAKI KOBAYASHI ${ }^{2}$, YUKA MATSUNAGA $^{2}$, SHOKO ISHIDA $^{2}$, \\ MINORU TAKATA ${ }^{1}$, OHTSURA NIWA ${ }^{1,2}$, EDUARDO A. PADLAN ${ }^{3}$ and ROLAND NEWMAN ${ }^{2}$ \\ ${ }^{1}$ Late effects Studies, Radiation Biology Center, Kyoto University, Yoshida-Konoe-cho, Sakyo-ku, Kyoto 606-8501; \\ ${ }^{2}$ Toyomi Laboratory, BioMedics Japan, Inc., 4-18 Toyomi, Chuo-ku, Tokyo 104-0055, Japan; \\ ${ }^{3}$ Marine Science Institute, College of Science, University of the Philippines, Quezon City 1101, Philippines
}

Received September 7, 2010; Accepted October 26, 2010

DOI: $10.3892 /$ ijo.2010.864

\begin{abstract}
Rituximab (chimeric anti-CD20 mAb) is currently used in the treatment of B-NHL and B cell malignancies, alone or in combination with chemotherapy. However, subsets of patients do not initially respond and/or develop resistance to additional treatments. Hence, there is a need to develop more effective anti-CD20 mAbs that may improve clinical response. BM-ca is a novel humanized anti-CD20 $\mathrm{mAb}$ that was tested against several B-NHL cell lines and was compared to several anti-CD20 mAbs (Rituximab, ofatumumab, 2H7, B1 and B-Ly1). BM-ca was shown to strongly induce both homotypic cell aggregation and redistribution of CD20 to membrane lipid rafts. BM-ca was also able to induce programmed cell death (apoptosis) without the need for cross-linking and demonstrated potent complement-dependent cytotoxicity (CDC). BM-ca was more cytotoxic than rituximab even in malignant $B$ cells expressing low amounts of membrane CD20. Type I antiCD20 mAbs typically induce minimal levels of homotypic cell aggregation and apoptosis but strong localization of CD20 to lipid rafts and potent CDC. Type II anti-CD20 mAbs typically exert the reverse activities. Noteworthy, BM-ca exhibits properties that are shared by both type I and type II anti-CD20 mAbs, which may reflect the recognition of a new CD20 epitope and/or exhibit different molecular signaling. Overall, the present data show that BM-ca is a novel anti-CD20 mAb that may be classified as a type I/II. The therapeutics efficacy of BM-ca awaits its use in clinical trials.
\end{abstract}

Correspondence to: Dr Michio Nishida, Late Effects Studies, Radiation Biology Center, Kyoto University, Yoshida-Konoe-cho, Sakyo-ku, Kyoto 606-8501, Japan

E-mail:minishida@aol.com

Key words: type I/IIanti-CD20 antibody, homotypic cell aggregation, CD20 redistribution to lipid rafts, apoptosis, C1q binding, B-NHL

\section{Introduction}

The use of rituximab as a single agent or in combination with cyclophosphamide, hydroxydaunorubicin, oncovin and prednisone (CHOP), is now a standard therapy for nonHodgkin's lymphoma (NHL) $(1,2)$. NHL patients do not always completely respond to rituximab therapy, however, and a subset of patients who initially responded become resistant or less responsive to further treatment (3). Despite a great number of reports, the in vivo mechanisms responsible to predict the clinical outcome in NHL after anti-CD20 treatment remain elusive. Complement-dependent cytotoxicity (CDC), antibody-dependent cellular cytotoxicity (ADCC) and apoptosis are all potential cytotoxic mechanisms, although it is unclear which mechanism is important in vivo. Redistribution of CD20 to membrane lipid rafts by anti-CD20 results in the clustering of antibody molecules and enhancing $\mathrm{C} 1 \mathrm{q}$ binding, the first step in the CDC induction mechanism (4). In addition to these above cytotoxic mechanisms, homotypic cell aggregation has been reported to be involved in antibody-induced cytotoxicity as well as the induction of apoptosis (5-7).

CD20 antibodies have been classified as type I or type II depending on their functional characteristics. Type I exhibits strong CDC activity but weak apoptosis whereas type II shows the opposite, i.e., weak CDC and strong apoptosis. Type I antibodies have the ability to redistribute CD20 to membrane lipid rafts whereas type II do not. Further, type I induce little homotypic cell aggregation whereas homotypic cell aggregation is significant for type II. Both type I and type II antibodies are equally effective in mediating ADCC. The differences observed between type I and type II are not explained by their affinity or isotype but most likely lie in their differences in epitope recognition. Rituximab (2B8), ofatumumab, ocrelizumab (2H7), 1F5, veltuzumab (A20) and AME-133V have been classified as type I antibodies and tositumomab (B1) and GA101 (B-ly1) as type II (8-10).

Cragg et al have reported that type II mAbs are superior to type I in depleting malignant B cells in mouse xenograft models, implying that ADCC and programmed cell death are the major in vivo mechanisms of cell killing as type II mAbs show weak CDC activity (10). Hence, an antibody capable of 
inducing strong ADCC in vitro may have a high initial activity in vivo and destroy circulating B cells by Fc receptor-bearing NK cells. However, NK cell activity is readily exhausted at high antibody concentrations and they require 'reloading' before they can resume their killing ability as Taylor and Lindorfer have pointed out (11). Differentiated macrophages in the liver (Kupffer cells) and spleen also express Fc receptors and can remove opsonized circulating tumor cells, although most of the ADCC activity in peripheral blood is mediated by NK cells (12). It is not clear whether NK cells or macrophages play a major role accessing and eliminating tumor cells sequestered in the lymph nodes and bone marrow in patients with B-NHL. Further it is not ascertained whether the mechanisms in human disease may be extrapolated from the mechanisms shown in animal models.

BM-ca is a humanized anti-CD20 mAb-derived from the murine mAb $1 \mathrm{~K} 1791$, which utilizes unique $\mathrm{VH}$ and $\mathrm{VL}$ germline genes and potent effector functions that inhibit certain intracellular survival pathways $(13,14)$. The present study was designed to investigate the following characteristics of BM-ca in comparison with several anti-CD20 mAbs, namely: i) binding to different tumor B cell lines; ii) direct induction of apoptosis; iii) homotypic cell aggregation; iv) redistribution of CD20 to membrane lipid rafts; and v) C1q binding and cell killing by CDC. The study was performed by comparing $\mathrm{BM}$-ca with the type I mAbs rituximab, ofatumumab and 2H7 and the type II mAbs B1 and B-ly1.

\section{Materials and methods}

Cells and antibodies. The human B cell lines, RC-K8 (histiocytic B cell lymphoma) and SU-DHL-4 (diffuse large cell, cleaved cell type B-NHL) were obtained from DSMZ GmbH (Braunschweig, Germany) and Raji and Ramos (Burkitt's lymphoma) were derived from JCRB (Riken Bioresource Center, Tsukuba, Japan). The following anti-CD20 mAbs, rituximab (chimeric) were obtained as follows: Zenyaku Kogyo (Tokyo, Japan), 2H7 (mouse) from GeneTex (Irvine, CA, USA), B1 (or tositumomab, mouse) from Beckman Coulter (Orange County, CA, USA) and B-ly1 (mouse) from Abcam (Cambridge, UK). B1 was provided for use after repurification by an affinity chromatography to remove sodium azide. Ofatumumab (human) was generated through reverse engineering of the DNA sequences described in US patent publication (US 2004/0167319 A1). Infliximab used as a negative control was obtained from Mitsubishi Tanabe Pharma (Osaka, Japan). Human mAb against hepatitis B virus surface antigen (HBs) was genetically engineered at BioMedics using DNA sequences in the public domain (NCBI: AF027159, AF027158). Human serum complement, C1q and C1q depleted human sera were obtained from Quidel (San Diego, CA, USA), Polyclonal rabbit anti-C1q antibody/FITC and propidium iodide were from DakoCytomation (Glostrup, Denmark) and Sigma (St. Louis, MO, USA), respectively. FITC conjugated goat anti-human IgG was from Invitrogen Japan (Tokyo, Japan).

Binding of anti-CD20 mAbs to different $B$ cells. The binding of BM-ca and rituximab to CD20-expressing B cell lines, RC-K8, SU-DHL-4, Ramos and Raji cells were examined to determine the differences in cell surface expression. FITC was conjugated to the anti-CD20 antibodies using a fluorescein labeling kit-NH2 and the provider's protocol (Dojindo Molecular Technologies, Kumamoto, Japan). Each antibody was conjugated directly to FITC. Cells were transferred to a $15 \mathrm{ml}$ tube in RPMI-1640 culture medium containing $10 \%$ fetal bovine serum (FBS) and centrifuged at $800 \mathrm{x} \mathrm{rpm}$ for 5 min. The supernatants were discarded, the pellets were washed once by PBS and then re-suspended in PBS containing $4 \%$ FBS (4\% FBS/PBS) at $1 \times 10^{6}$ cells $/ \mathrm{ml}$. A $100-\mu 1$ aliquot was placed into a $1.5 \mathrm{ml}$ tube and mixed with $100 \mu 1$ of FITClabeled antibody and incubated at room temperature for $30 \mathrm{~min}$ in dark. After washing twice with $4 \% \mathrm{FBS} / \mathrm{PBS}$, the cells were examined by flow cytometry (Guava EasyCyte Plus ${ }^{\mathrm{TM}}$, Millipore, Billerica, MA, USA) to calculate the mean of fluorescence intensity (MFI) and compare the differences in CD20 expression of each cell line.

Induction of apoptosis. The ability of BM-ca to induce apoptosis in target cells without secondary cross-linking agents was investigated by flow cytometry using the Annexin V/FITC and propidium iodine (PI) staining method (AnnexinV/FITC apoptosis detection kit, BD Biosciences, Franklin Lakes, NJ, USA). The type I mAbs rituximab, ofatumumab and $2 \mathrm{H} 7$ and the type II mAbs B1 and B-ly1 were compared using a negative control without mAb. RC-K8, Raji and SU-DHL-4 cells were used as target cells. Cells were maintained in RPMI-1640 with $10 \%$ FBS and sub-cultured for a further day at $2-4 \times 10^{5}$ cells $/ \mathrm{ml}$ prior to their use in the assay. The cells were incubated in duplicate tubes with $10 \mu \mathrm{g} / \mathrm{ml}$ test or control mAbs. After incubation for $24 \mathrm{~h}$, the cells were pelleted by centrifugation, washed and re-suspended in binding buffer (10 mM HEPES, pH 7.4; $140 \mathrm{mM} \mathrm{NaCl} ; 2.5 \mathrm{mM}$ $\mathrm{CaCl}_{2}$ ) and stained with Annexin V/FITC and PI according to the manufacturer's specification (BD BioSciences, San Jose, CA, USA). All samples were applied to a flow cytometer Guava EasyCyte Plus ${ }^{\text {TM }}$ and analyzed by CytoSoft 5.3 software to calculate the ratio of apoptotic cells (FITC) to dead cells (PI). All raw data were revised by subtracting the value from negative control (anti-HB mAb treated) gained in the same experiment.

Homotypic cell aggregation. BM-ca was tested for its ability to induce homotypic cell aggregation and compared to rituximab, ofatumumab and $2 \mathrm{H} 7$ type I mAbs and B1 and B-ly1 type II mAbs. RC-K8, Ramos and SU-DHL-4 cells were used as target cells and a sample without antibody was used to determine the background. Cells were cultured in RPMI-1640 with 10\% FCS until reaching a density of $2 \times 10^{6}$ cells $/ \mathrm{ml}$. The concentration of each mAb was adjusted to $2 \mu \mathrm{g} / \mathrm{ml}$ in $500 \mu 1$ of RPMI-1640 solution. Cell suspension $(100 \mu \mathrm{l})$ was pipetted into each well of a 48-well micro-titer plate and then mAb solution was added to each well and mixed. The plate was incubated at $37^{\circ} \mathrm{C}$ for $4 \mathrm{~h}$ and mixed gently, after which samples were collected from each well and examined. The aggregates were classified into three different groups, small clusters (3-5 cells, 4 cells on average), medium clusters (6-20 cells, 12 cells on average) and large clusters (>20 cells). The level of aggregation was determined by the following formula: Cell aggregation (\%) $=[(4 \mathrm{x}$ number of small cluster $)+(12 \mathrm{x}$ number of median 
cluster + accumulated cell number of large cluster)/total cell number] x 100 .

Redistribution of CD20 to membrane lipid rafts following binding by $m A$ bs. BM-ca was tested for its ability to redistribute CD20 to membrane lipid rafts and compared to rituximab, ofatumumab, 2H7, B1 and B-ly1 using the method described by Cragg et al (15) RC-K8, Raji and SU-DHL-4 cells were used as target cells and cultured in RPMI-1640 (Sigma) with $10 \%$ fetal bovine serum (FBS). Cells were washed with PBS, re-suspended in $4 \% \mathrm{FBS} / \mathrm{PBS}$ and adjusted to $2 \times 10^{6}$ cells $/ \mathrm{ml}$. Antibodies were diluted in PBS $(500 \mu \mathrm{g} / \mathrm{ml})$ and labeled with FITC using the same method described in section 2 of Materials and methods. Cell suspension $(250 \mu 1)$ was added to $5 \mu 1$ of diluted FITC labeled mAb and incubated for $15 \mathrm{~min}$ at room temperature. The antibody-bound cells were washed using ice cold PBS with 4\% FBS and re-suspended into two vials (100 $\mu 1$ each). One vial was maintained on ice to allow calculation of $100 \%$ surface antigen level and the other was treated with $5 \mu \mathrm{l}$ of Triton X-100 solution (10\%) for $15 \mathrm{~min}$, mixed gently and maintained on ice to determine the population of CD20 antigens remaining in the insoluble lipid raft fraction. Cells were maintained at $4^{\circ} \mathrm{C}$ throughout the remainder of the assay, washed once in PBS with 4\% FBS, re-suspended in a $500 \mu 1$ tube, and then assessed by flow cytometry. Cells with or without Triton X-100 treatment were compared using the mean fluorescent intensity (MFI) measured by flow cytometry. Percentage insolubility was calculated by the formula: Insolubility $(\%)=[\mathrm{MFI}$ (with Triton X-100]/MFI (without Triton X-100) ] x 100.

Clq binding and cell killing. Binding of the C1q complex to mAbs bound to CD20 on target cells was assessed by a cell based assay using flow cytometry (16). BM-ca was tested and compared to the type I antibodies rituximab and ofatumumab. RC-K8 and SU-DHL4 were selected as target cells which were cultured in RPMI-1640 with $10 \%$ FBS, washed and resuspended in RPMI-1640 media, supplemented with $1 \mathrm{mM}$ $\mathrm{MgCl}_{2}$ and $1 \mathrm{mM} \mathrm{CaCl}$, and adjusted to $5 \times 10^{6}$ cells $/ \mathrm{ml}$. The test $\mathrm{mAbs}$ and $\mathrm{C} 1 \mathrm{q}$ complex were added to the culture at a final $\mathrm{mAb}$ concentration of 10 and $40 \mu \mathrm{g} / \mathrm{ml}$, respectively, and reacted for $10 \mathrm{~min}$ at $37^{\circ} \mathrm{C}$. Various amounts of $\mathrm{C} 1 \mathrm{q}$ depleted human sera were then added to the cultures to a final concentration of $0,1,5$ and $20 \%$. The cultures were harvested, washed with ice-cold 4\% FBS/PBS, and treated with anti-C1q FITC-labeled antibodies and PI on ice for $30 \mathrm{~min}$ before examination by a flow cytometry Guava EasyCyte Plus. The results were analyzed by CytoSoft 5.3 software (Millipore) to measure the $\mathrm{C} 1 \mathrm{q}$ binding (FITC) and cell death (PI). The time course of $\mathrm{C} 1 \mathrm{q}$ binding and cell killing were also examined simultaneously at 5- and 30-min time-points after adding C1q-depleted human serum to a final concentration of $0,1,5$ and $20 \%$.

\section{Results}

Different surface CD20 distribution patterns among B cells expressed by $B M-c a$ and rituximab. The surface binding of BM-ca (Fig. 1a) and rituximab (Fig. 1b) on RC-K8, SUDHL-4, Ramos and Raji cell lines was examined by flow cytometry. The mean fluorescence intensity (MFI) of antibodybinding to each B cell line by BM-ca and rituximab showed differences in membrane CD20 expression. Rituximab and BM-ca showed similar binding patterns on each cell line with the highest level of CD20 expression found on SU-DHL-4 cells. The MFI for SU-DHL-4 cells was approximately twice that of RC-K8 and more than twice that of Ramos and Raji cells.

BM-ca-induced apoptosis. The Annexin V/PI method was used to assess the ability of various anti-CD20 mAbs to induce cell death by apoptosis as described in Materials and methods. Fig. 2a shows a representative flow analysis of the various subsets of cells following treatment, namely live cells, necrotic death, apoptotic death and apoptotic live. The various antibodies were compared and the data are represented in bar graphs for the three cell lines studied, namely, RC-K8 (Fig. 2b) Raji (Fig. 2c) and SU-DHL-4 (Fig. 2d). The type I antibodies, rituximab, ofatumumab and $2 \mathrm{H} 7$ induced little or no apoptosis in either RC-K8 or Raji cells but were active against SU-DHL-4 cells which had a much higher level of CD20 expression (Fig. 1). The ability of BM-ca to induce apoptosis was lower than B1 but more potent than B-Ly1 using RC-K8 and Raji as targets however, a similar level of death and apoptosis was observed among all antibodies when SU-DHL-4 cells were used. It is noteworthy, however, that the degree of apoptosis induced by BM-ca and type II mAbs was different from that of type I mAbs after $24 \mathrm{~h}$ of $\mathrm{mAb}$ treatment. Rituximab and ofatumumab type I antibodies induced early apoptotic events but most of the cells remained alive (Annexin V-positive but PI-negative), whereas BM-ca and type II more effectively led to complete apoptotic cell death (Annexin Vpositive and PI-positive). These findings suggest that BM-ca and type II mAbs are still more potent in inducing apoptotic death, even in high CD20 expressing cells such as SU-DHL-4. Type I antibodies appeared to induce the early event associated with apoptosis but were not able to sustain the effect to drive the cells completely along the apoptotic cell death pathway.

BM-ca induces strong homotypic cell aggregation. Exposure to $\mathrm{BM}$-ca resulted in extremely strong homotypic cell aggregation in all cells tested, i.e. RC-K8 (Fig. 3a), Ramos (Fig. 3b) and SU-DHL-4 cells (Fig. 3c), compared to the other type I and type II antibodies. The pattern of aggregation with RC-K8 and Ramos cells was similar for the different antibodies with type I antibodies showing little aggregation and type II showing a greater level of aggregation, although BM-ca always showed the greatest homotypic aggregation activity (Fig. 3a and b). When SU-DHL-4 cells were used, type I mAbs showed a higher level of aggregation (Fig. 3c). By comparison, antibody 2H7 did not induce aggregation in any of the cell lines tested.

Redistribution of CD20 to membrane lipid rafts. Redistribution of CD20 to membrane lipid rafts is important in providing the appropriate configuration of antibody Fc regions for the optimal recruitment of $\mathrm{Clq}$ and the triggering of the classical pathway of CDC. The analyses were performed with the RC-K8 (Fig. 4a), Raji (Fig. 4b), and SU-DHL-4 (Fig. 4c) cell 

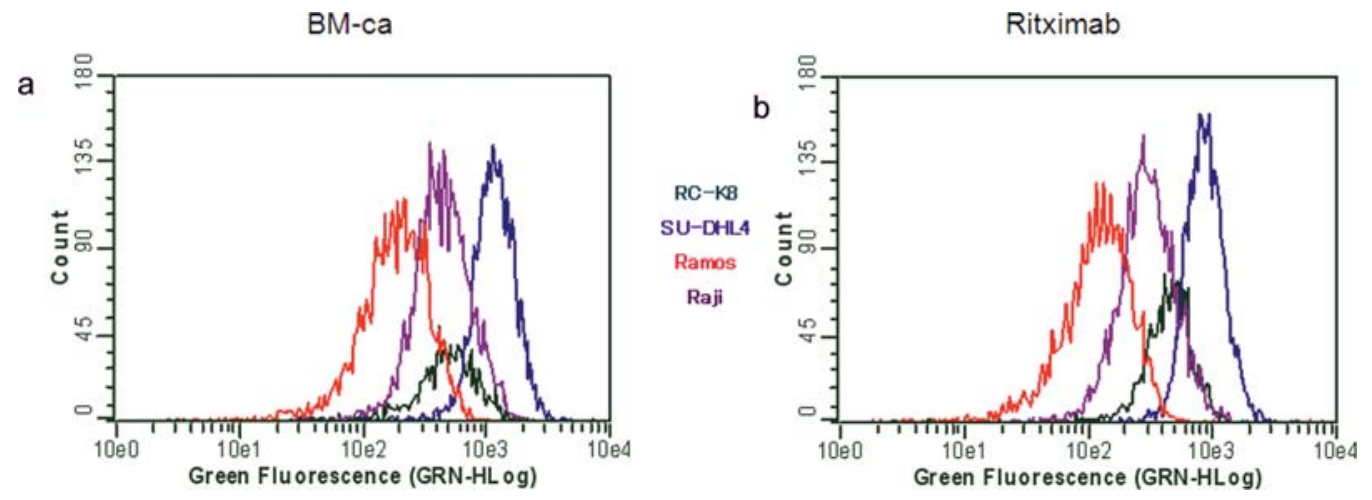

Figure 1. Binding of BM-ca and rituximab to various CD20-expressing B-NHL cell lines. The cell lines RC-K8, SU-DHL-4, Ramos and Raji were treated each with either BM-ca (a) or rituximab (b) and analyzed by flow cytometry for the expression of CD20 and the mean flurorescent intensities were recorded as described in Materials and methods.

a

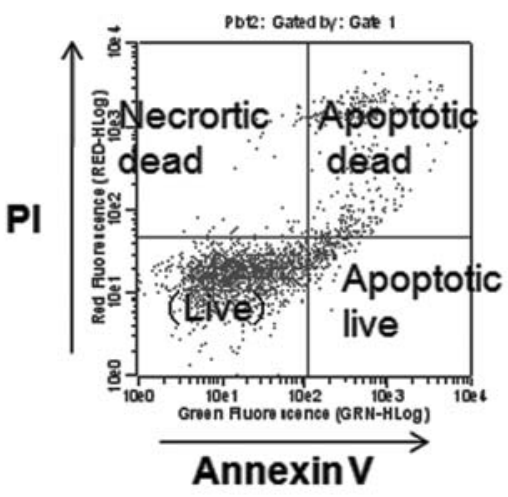

Total dead/apoptotic: necrotic dead+apoptotic dead+apoptotic live

C

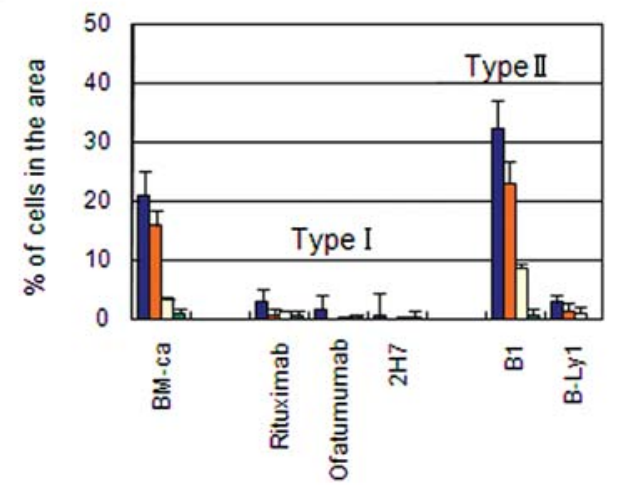

b

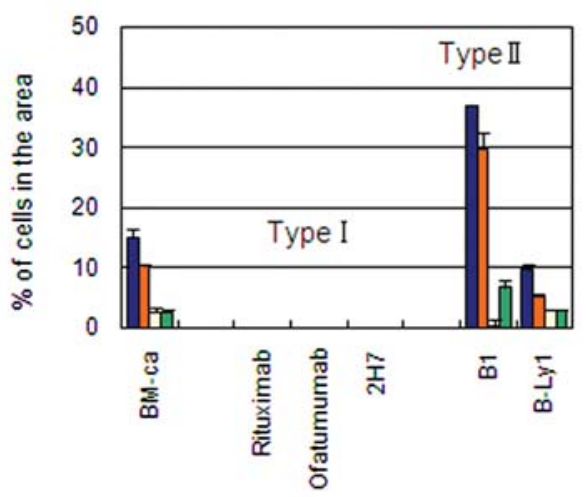

Figure 2. Analysis of the apoptotic activity of various anti-CD20 mAbs on B-NHL cells. The apoptotic activity was assessed by the Annexin V/PI staining method described in Materials and methods. (a) Representative for the various properties of the cells analyzed by flow cytometry. The data for the cell lines RC-K8 (b) Raji cells (c), and SU-DHL-4 (d) are shown. The data represent the mean \pm SD of two independent experiments performed with each cell line. Blue bar, total dead/apoptotic; orange bar, apoptotic dead; yellow bar, apoptotic, live; green bar, necrotic dead.

lines using several anti-CD20 mAbs. BM-ca as well as the type I mAbs rituximab and ofatumumab induced significant redistribution of CD20 to lipid rafts after binding, as shown by $>60 \%$ of Triton X-100 insoluble CD20 antigens remaining in the insoluble lipid raft fraction. On the other hand, relocation by type II mAbs was extremely low in $2 \mathrm{H} 7$, however, even though d

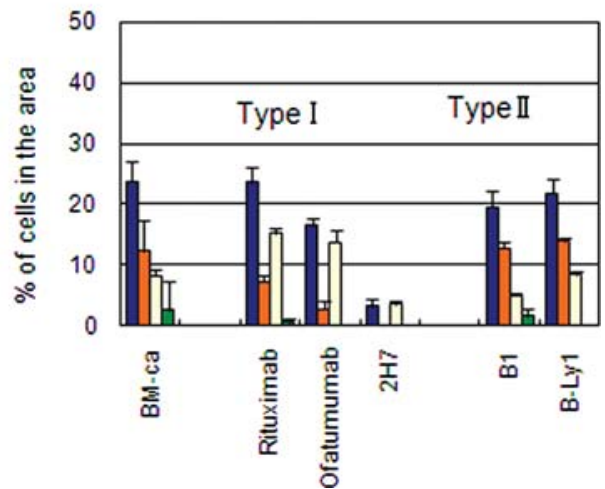

$2 \mathrm{H} 7$ has been previously classified as a type I antibody. It demonstrated a moderate level of CD20 redistribution.

Clq binding and cell killing. C1q binding to antibody is the initial step in complement-dependent cell killing (CDC). Binding of C1q complex to BM-ca, rituximab and ofatumumab 
a

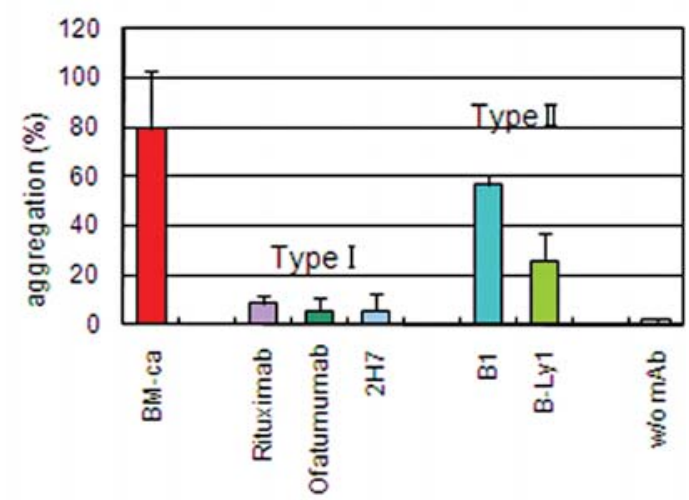

b

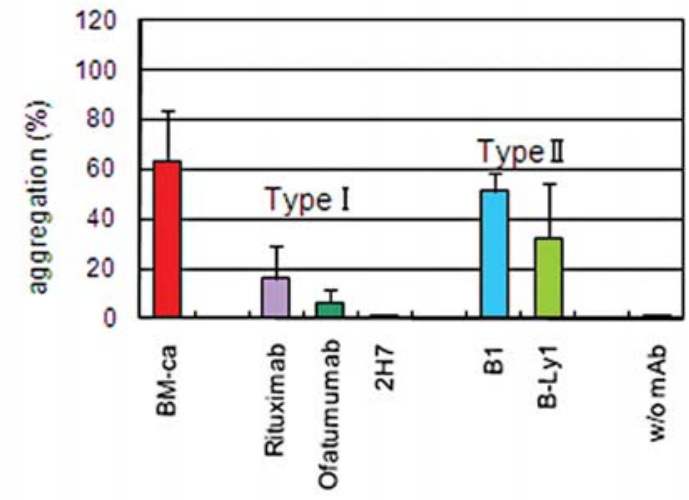

C

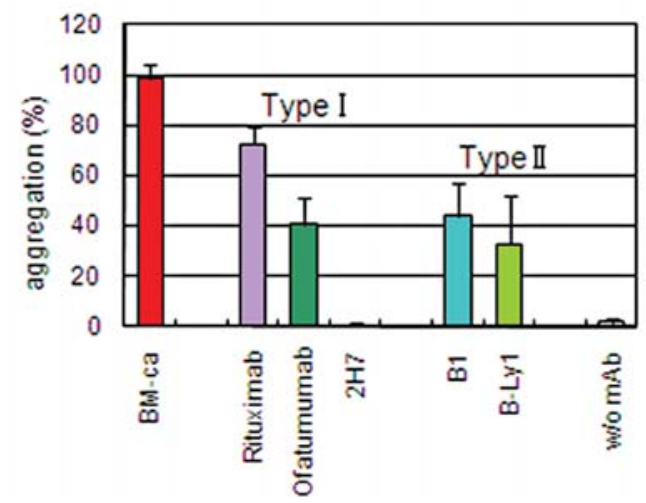

Figure 3. Induction of homotypic aggregation by various anti-CD20 mAbs. The RC-K8 (a), Ramos (b), SU-DHL-4 (c) cells were treated by various anti-CD20 mAbs and tested for homotypic aggregation as described in Materials and methods. The data are presented as \% aggregation and are the mean \pm SD of two independent experiments.

in the absence of other complement components was evident, as shown in the top line of Fig. 5a and b whereby no C1qdepleted human serum was added. The MFI values for BM-ca, rituximab and ofatumumab were 630, 566 and 939, respectively, for RC-K8 cells (top line of Fig. 5a), and 1232, 1027 and 1267 , respectively, for SU-DHL-4 cells (top line of Fig. 5b). The addition of $20 \%$ C1q-depleted human serum as a source of other complement components was sufficient for BM-ca and ofatumumab to induce significant cell death in $\mathrm{RC}-\mathrm{K} 8$, although rituximab remained ineffective, as shown in the fourth line of Fig. 5a. For SU-DHL-4 cells 5\% C1q depleted human serum was sufficient to induce almost complete cell killing by all the mAbs as shown in the third a

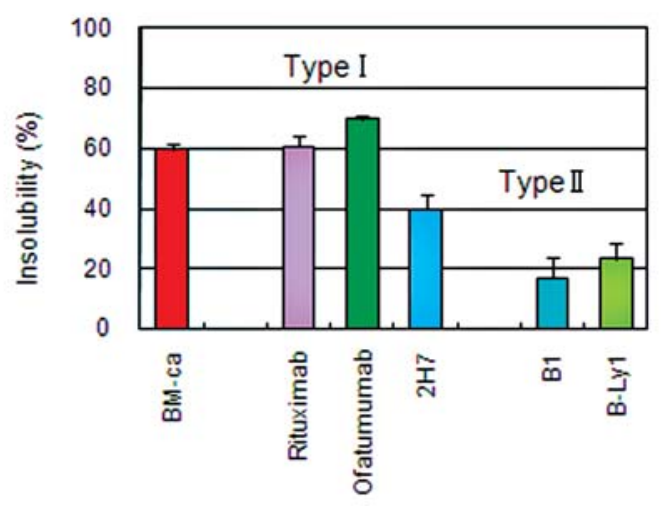

b

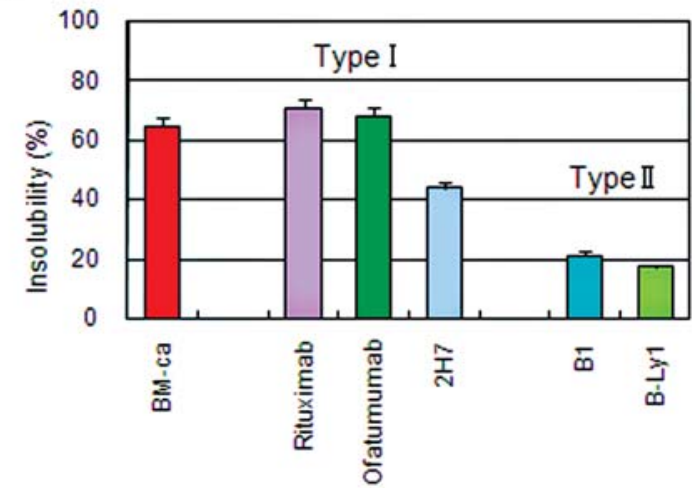

C

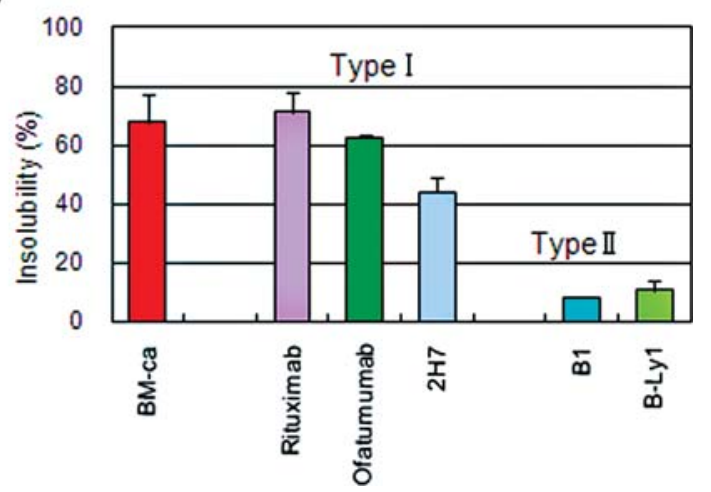

Figure 4. CD20 redistribution to membrane lipid rafts by various anti-CD20 antibodies. The ability of BM-ca, rituximab, ofatumumab, 2H7, B1 and B-Ly1 mAbs to redistribute CD20 in lipid drafts were determined as described in Materials and methods. The data are presented as mean \pm SD of three independent experiments for both RC-K8 and SU-DHL-4 and two for Raji. The data are shown for RC-K8 cells (a), Raji (b), and SU-DHL-4 (c). The cells with or without Triton X-100 treatment were compared using the mean fluorescent intensity measured by flow cytometry as described in Materials and methods.

and fourth lines of Fig. 5b. Rituximab-induced CDC activity was similar to the other antibodies with SU-DHL-4 cells. The maximum cytotoxic effect occurred within 5 min of $\mathrm{C} 1 \mathrm{q}$ binding and after 30 min the cell killing did not exceed the 5-min values (Fig. 6).

\section{Discussion}

Evidence is presented here that BM-ca possesses biological properties that are associated with both type I and type II 
a

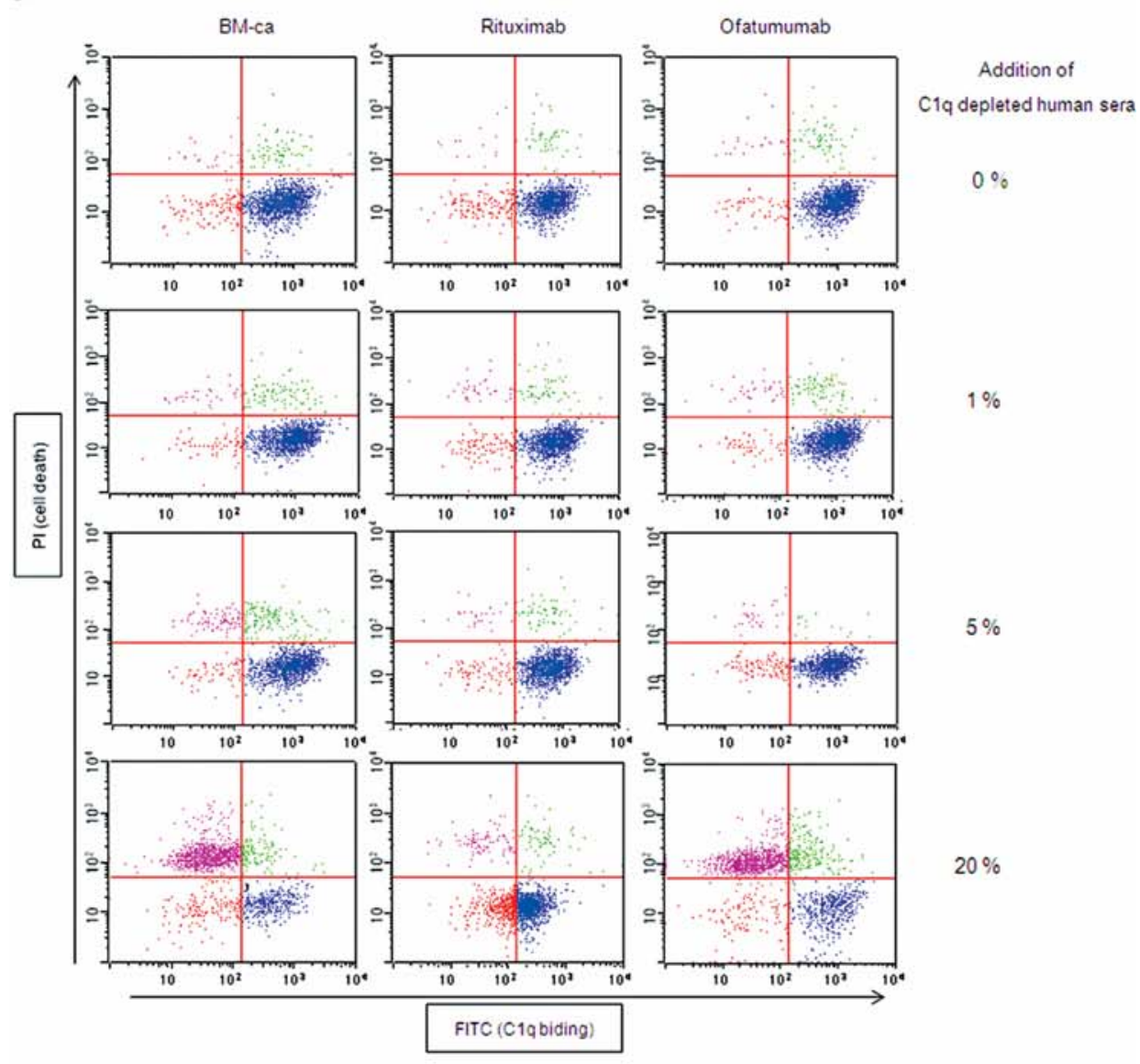

b
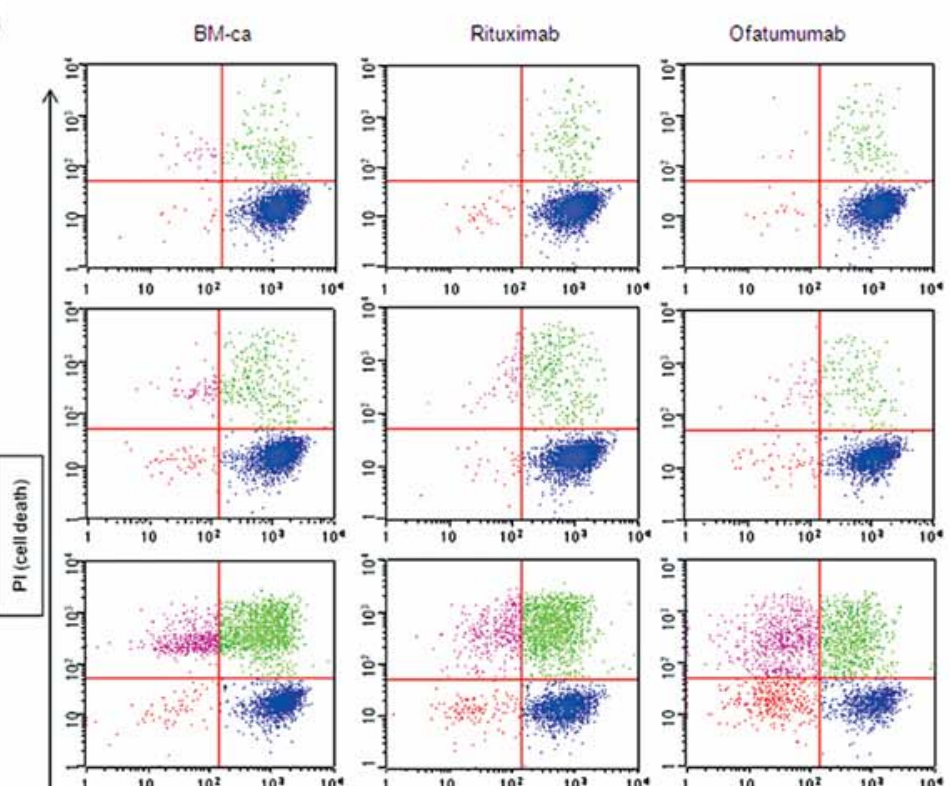

Addition of

C1q depleted human sera

$0 \%$
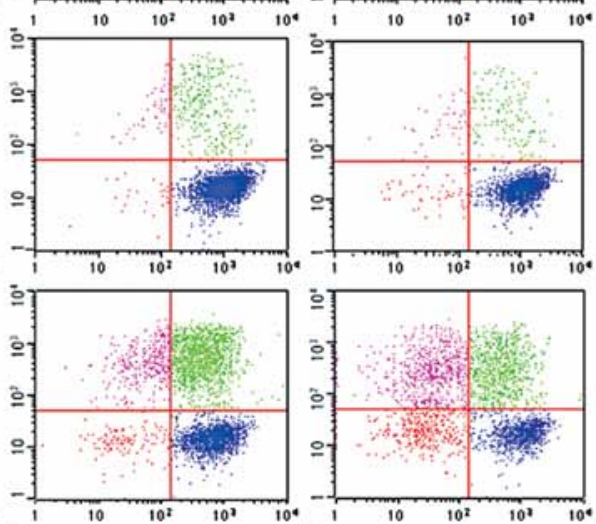

$1 \%$
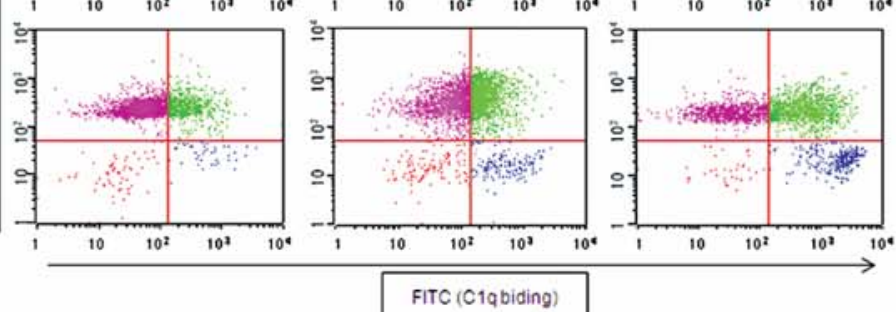

$5 \%$

$20 \%$

Figure 5. Binding of C1q by anti-CD20 mAbs and cell killing. The C1q binding and cell killing were determined as described in Materials and methods. The data in (a) and (b) represent analyses of RC-K8 and SU-DHL-4, respectively. The three columns show the results of BM-ca, rituximab, and ofatumumab, respectively, and the first to the fourth rows show the effect of adding different percentages of C1q-depleted human serum from 0 to $20 \%$. C1q binding to rituximab or ofatumumab in the absence of other components are shown in the top line of (a) and (b). 


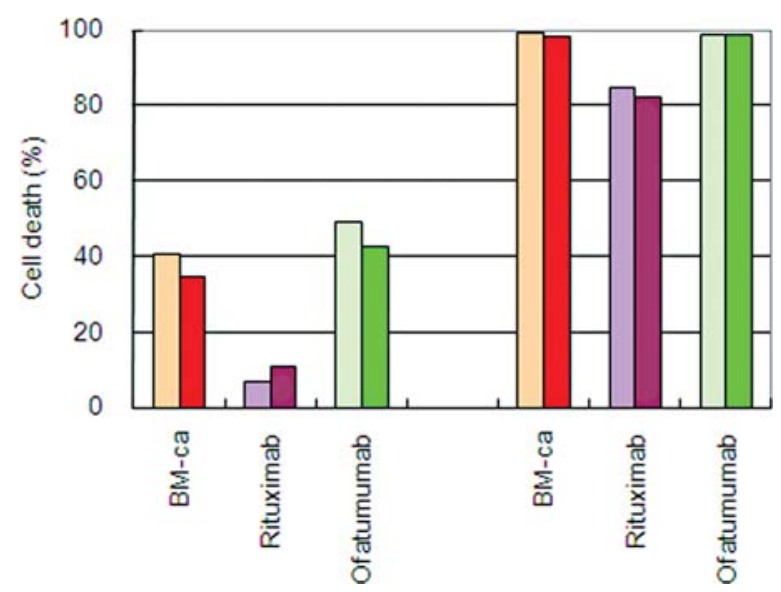

Figure 6 . The time course of complement-induced cytotoxicity by each $\mathrm{mAb}$ of RC-K8 (left-hand side bars) and SU-DHL-4 (right-hand side bars) is shown. The percentages of cell death with each $\mathrm{mAb}$ are shown after $5 \mathrm{~min}$ (left bar of each $\mathrm{mAb}$ ) and after 30 min (right bar of each $\mathrm{mAb}$ ).

anti-CD20 mAbs. The various findings with BM-ca and the comparison with the various type I and type II anti-CD20 $\mathrm{mAbs}$ are summarized in Table I. The induction of apoptosis by BM-ca, in the absence of cross linking, was significant and much greater than the weak apoptosis induced by rituximab. The apoptotic activity is enhanced by cross-linking for all antibodies although the differences among the antibodies following cross-linking are not significant (17). The level of cytotoxicity observed in the apoptosis assays was not as high as those achieved by $\mathrm{CDC}$ or ADCC. The direct apoptosis achieved by BM-ca may be beneficial due to its longer time span and, presumably, may trigger a series of intracellular signaling pathways that induce cell death and inhibit cell survival. Vega et al (14) reported that BM-ca was superior to rituximab in the inhibition of phosphorylation of p38 and the transcription factor NF- $\mathrm{KB}$ which led to the inhibition of the anti-apoptotic gene product Mcl-1 and the induction of the pro-apoptotic gene product Bax. Furthermore, they showed that BM-ca was a stronger inducer of Raf-1 kinase inhibitor protein (RKIP), a metastasis suppressor and immune surveillance cancer gene product $(9,14)$.

Type II antibodies were generally similar or superior to BM-ca at inducing apoptosis although the extent depended on the cell type and the level of CD20 expression. It is generally assumed that apoptosis induction begins with the cross-linking or reorientation of antigen to trigger a series of intracellular signals. Therefore, the differences between type I, type II and BM-ca antibodies probably reflect the localization of epitopes on the CD20 molecule and the ability by which different antibodies can cause a shift in conformation.

The ability of anti-CD20 antibodies to induce homotypic cell aggregation is a phenomenon that is poorly interpreted as the simple physical bridging of cells by antibody. Type I antibodies typically do not induce homotypic cell aggregation whereas type II and BM-ca are quite potent inducers of this phenomenon despite similar affinities of these antibodies. Kansas and Tedder showed that cellular energy was required for homotypic cell aggregation and was influenced by the

Table I. Comparison of the properties of anti-CD20 mAbs.

\begin{tabular}{|c|c|c|c|c|c|c|c|}
\hline & & \multicolumn{3}{|c|}{ Homotypic cell aggregation (\%) } & \multicolumn{3}{|c|}{ Apoptosis (\%) } \\
\hline & & $\mathrm{RC}-\mathrm{K} 8$ & Ramos & SU-DHL-4 & $\mathrm{RC}-\mathrm{K} 8$ & Ramos & SU-DHL-4 \\
\hline BM-ca & Type I/II & 80 & 60 & $\leq 100$ & 20 & 20 & 20 \\
\hline Rituximab & Type I & $<10$ & $<\mathbf{2 0}$ & 70 & 0 & 0 & 20 \\
\hline Ofatumumab & Type I & $<10$ & $<\mathbf{1 0}$ & 40 & 0 & 0 & 20 \\
\hline $2 \mathrm{H} 7$ & Type I & $<10$ & $\mathbf{0}$ & $\mathbf{0}$ & 0 & 0 & 0 \\
\hline B1 & Type II & 60 & 50 & 40 & 40 & 40 & 20 \\
\hline \multirow[t]{3}{*}{ B-ly1 } & Type II & 20 & 30 & 30 & 10 & 0 & 20 \\
\hline & & \multicolumn{3}{|c|}{ CD20 redistribution to lipid rafts $(\%)$} & \multicolumn{3}{|c|}{$\mathrm{CDC}(\%)$} \\
\hline & & $\mathrm{RC}-\mathrm{K} 8$ & Ramos & SU-DHL-4 & $\mathrm{RC}-\mathrm{K} 8$ & Ramos & SU-DHL-4 \\
\hline BM-ca & Type I/II & 60 & 60 & 70 & 40 & $\leq 100$ & $\leq 100$ \\
\hline Rituximab & Type I & 60 & 70 & 70 & 10 & $\leq 100$ & 80 \\
\hline Ofatumumab & Type I & 70 & 70 & 60 & $40-50$ & $\leq 100$ & $\leq 100$ \\
\hline $2 \mathrm{H} 7$ & Type I & 40 & 40 & 40 & nt & nt & nt \\
\hline B1 & Type II & 20 & 20 & 10 & $\mathrm{nt}$ & $\mathrm{nt}$ & $\mathrm{nt}$ \\
\hline B-ly1 & Type II & 20 & 20 & 10 & nt & nt & nt \\
\hline
\end{tabular}

The numbers for apoptosis and CDC are percentages of cell death. Homotypic cell aggregation is expressed as the percentage of aggregates and CD20 distribution to lipid rafts as percentage of insolubility. The results of CDC with Ramos cells refer to our unpublished experiments. Bold, values that are not considered significant in the particular assay. $\leq 100$, close to $100 \%$; <10, very little; nt, not tested. 
antigenic epitope rather than the mAb isotype or affinity (18). Further, Ivanov et al demonstrated that certain antibodies could cause the migration of mitochondria to the site of cellcell contact and with the subsequent induction of lysosomal leakage (5). BM-ca showed strong induction of homotypic cell aggregation in all three cell lines tested. Our findings demonstrate that type I mAbs showed weak homotypic cell aggregation activity and the two type II antibodies tested showed moderate activity, but less than BM-ca. In the case of the SU-DHL-4 cell line, all antibodies (type I and II), with the exception of $2 \mathrm{H} 7$, showed significant levels of homotypic cell aggregation, suggesting that when the expression of CD20 on the cell surface is high, certain differences between type I and type II antibodies are minimized. The level of homotypic cell aggregation did not directly correlate with the ability to induce apoptosis. For example, BM-ca was able to induce homotypic cell aggregation in RC-K8 and Raji cells more efficiently than B1 but the reverse was true for the induction of apoptosis.

The ADCC effector mechanism is not discussed in this report for the unavailability of the humanized versions of type II mAbs. However, BM-ca was compared with type I mAbs rituximab and ofatumumab (2F2) for its ability to induce ADCC and our previous report showed little difference among these antibodies (13). As ADCC mainly depends on the binding of the immunoglobulin Fc to the Fc $\gamma$ RIIIa (CD16), there may be minimal differences between mAbs of the same isotype, except for those in which the Fc region carbohydrate structure has been modified to remove fucose $(19,20)$.

It is not clear which functions are of primary importance for the in vivo clinical benefit of anti-CD20 mAbs. Mouse models using either human tumor xenografts or transgenic models have given conflicting results that may reflect the differences between human tumor cells and transgenic cells $(9,21)$. Minard-Colin et al however, have concluded that $\mathrm{Fc}: \mathrm{FcR}$ interactions are important in the in vivo mouse models (22). There appears to be little support for CDC being an important in vivo mechanism, although this conclusion is mainly inferential and is still controversial $(23,24)$. BM-ca has been reported to be more potent than rituximab in the chemosensitization of resistant B-NHL to CDDP-apoptosis (14). This may be the result of the ability of the antibody to reduce the anti-apoptotic threshold responsible to sensitize the cells to drug-induced apoptosis (25). Comparison to type II antibodies is a little more complex. The type II mAb B1 induced stronger apoptosis than BM-ca but weaker CDC activity, although BM-ca was superior to another type II mAb, B-ly1.

Beers et al reported that type II mAbs outperformed type I mAbs in B cell depletion in mouse xenograft models and recent experiments using CD20 transgenic mice have concluded that Fc:FcR interactions play a major role in both type I and type II antibodies $(10,22)$. The reason why type II antibodies should be superior is somewhat unclear. The authors concluded that depending on the model and whether malignant or normal B cells are used, different results and mechanisms may come into play. There have been no reports, thus far, on any type II mAbs in the clinic, with the exception of B1 conjugated to a radio-isotope (Bexxar) and GA101 which has a modified Fc carbohydrate structure $(26,27)$.
The findings in this report generally support previously reported data on the segregation between the activities of type I and type II antibodies. The exception seems to be the cell line SU-DHL-4 which, when tested, appears to decrease the differences between the activities of type I and type II antibodies. This finding with SU-DHL-4 may be due to the much higher level of CD20 expression on these cells, which could function to amplify certain minor activities seen on other cell lines. It is not clear whether SU-DHL-4 cells are or not representative of the in vivo situation with either normal or lymphoma cells. In addition to CD20 expression levels, it is possible that the antibodies signal SU-DHL-4 cells differently from their signaling of other cell lines.

Clearly, the delineation of the important in vivo mechanism of anti-CD20 mAbs is not simple and varies with the systems used, the cell types, the levels of CD20 expression and cell signaling. Nevertheless, the present data clearly show that BM-ca possesses both sets of biological properties that are usually segregated into either type I or type II activity, i.e., high level of CD20 redistribution to the lipid rafts, homotypic cell aggregation and apoptosis induction (Table I). Although the division of anti-CD20 into type I and type II may be an empirical classification, it likely reflects the binding to different epitopes on the CD20 molecule. Cragg et al hypothesized that type I mAbs lead CD20 tetramers to adopt an open type of configuration that is associated with an increased calcium flux whereas type II induce a more 'closed' type configuration $(9,27)$. BM-ca exhibits properties of both type I and type II and, therefore, it is unclear whether the CD20 epitope to which it binds fits neatly into one of these two alternatives or binds to a new class of epitope that is different from either type I and type II. The neat division of epitopes into type I or type II may simply reflect the antibodies that are currently available which are all derived from the same or similar germline genes. There may in fact be a continuum of epitopes and the rarer configurations may be missed because of the immunization protocol used.

We have previously reported that $1 \mathrm{~K} 1791$, the murine parent of BM-ca, was derived from the germline VH9 family and Vк19/28 family, which is different from any other reported murine-derived anti-CD20 mAbs published so far. In all other cases of murine-derived mAbs, the heavy chain $\mathrm{V}$ region genes were derived from the VH1 family genes $(13,28)$. These include 2B8, 2H7, 1F5, A20, B1 and B-Ly1 and most of other cases of the light chain $\mathrm{V}$ region genes were from $\mathrm{V \kappa}_{\kappa} 4 / 5$ family genes except for B-Ly1 which was Vк24/25. The more unusual immunoglobulin germline gene family used by BM-ca (1K1791) may recognize epitopes not seen by the VH1 family and we have previously concluded that the BM-ca epitope was conformational and highly susceptible to changes in tertiary structure. As a result of the unique biological properties of BM-ca, we suggest that BM-ca may be classified as type I/II anti-CD20 mAb.

The phenomenon of relapse and acquired resistance following rituximab treatment is a serious concern because of the subsequent lower response rate achieved after further treatment. This has been reported to be as low as $40 \%$ of those patients that initially responded to rituximab (5). The mechanism of such resistance has not been fully elucidated, however, a number of reports on the potential mechanisms 
of resistance after rituximab mono-therapy or R-CHOP combination therapy have been suggested. These include loss or down-regulation of CD20 membrane expression, histological transformation and up-regulation of anti-apoptotic molecules including bcl-2 (29-32). We are currently examining whether BM-ca, alone or in combination with chemotherapy, exerts an activity, not seen with rituximab, on rituximabresistant B-NHL cell lines.

In conclusion, our studies demonstrate the unique properties observed by BM-ca in comparison with several type I and type II antibodies. BM-ca is shown to exhibit activities shared by both type I and type II. Such activities may provide BM-ca with a potential novel therapeutic activity in B-NHL. The superiority of BM-ca awaits its examination in clinical trials for its therapeutic activity.

\section{Acknowledgements}

We acknowledge Dr Benjamin Bonavida for assisting in the revision and advising in the preparation of this manuscript. We also acknowledge Drs Akira Fujimori and Ryuichi Okayasu at the National Institute of Radiological Science for advising in our experiments. We thank BioMedics Japan, Inc. for the funding support. The assistance of Daphne Liang in the preparation of this manuscript is greatly appreciated.

\section{References}

1. Grillo-Lopez AJ, Dallaire BK, McClure A, Weaver R, Varns C, Wei A, Allen R, Lee D, Shen D, Leonard J, Multani P and White CA: Monoclonal antibodies: a new era in the treatment of non-Hodgkin's lymphoma. Curr Pharm Biotechnol 2: 301-311, 2001.

2. Coiffier B: Monoclonal antibodies in the management of newly diagnosed, aggressive B-cell lymphoma. Curr Hematol Rep 2: 23-29, 2003

3. Davis TA, Grillo-López AJ, White CA, McLaughlin P, Czuczman MS, Link BK, Maloney DG, Weaver RL, Rosenberg J and Levy R: Rituximab anti-CD20 monoclonal antibody therapy in non-Hodgkin's lymphoma: safe and efficacy of re-treatment. J Clin Oncol 18: 3135-3143, 2000

4. Walport MJ: Complement. First of two part. N Eng J Med 344: 1058-1066, 2001.

5. Ivanov A, Beers SA, Whalshe CA, Honeychurch J, Alduaji W, Cox KL, Potter KN, Potter KN, Murray S, Chan CHT Klymenko T, Erenpreisa J, et al: Monoclonal antibodies directed to CD20 and HLA-DR can elicit homotypic adhesion followed by lysosome-mediated cell death in human lymphoma and leukemia cells. J Clin Invest 119: 2143-2159, 2009

6. Jazirehi AR, Huerta-Yepez S, Cheng G and Bonavida B: Rituximab (chimeric anti-CD20 monoclonal antibody) inhibits the constitutive nuclear factor-\{kappa\}B signaling pathway in non-Hodgkin's lymphoma B-cell lines: role in sensitization to chemotherapeutic drug-induced apoptosis. Cancer Res 65: 264-276, 2005

7. Wobser M, Voigt H, Eggert AO, Houben R, Kauczok CS, Brocker EB and Becker JC: Bcl-2 expression in rituximab refractory cutaneous B-cell lymphoma. Br J Cancer 96: 1540-1543, 2007.

8. Cragg MS and Glennie MJ: Antibody specificity controls in vivo effector mechanisms of anti-CD20 reagents. Blood 103: 2738-2743, 2004.

9. Beers SA, Chan CHT, French RR, Cragg MS and Glennie MJ: CD20 as a target for therapeutic type I and II monoclonal antibodies. Semmin Hematol 47: 107-114, 2010.

10. Beers SA, Chan CT, James S, French RR, Attfield KE, Brennan CM, Ahuja A, Shlomchik MJ, Cragg MS and Glennie MJ: Type II (tositumomab) monoclonal antibody out per performs type I (rituximab-like) reagents in B-cell depletion regardless of complement activation. Blood 112: 4170-4177, 2008 .
11. Taylor RP and Lindorfer MA: Immunotherapeutic mechanisms of anti-CD20 monoclonal antibodies. Curr Opin Immunol 20: 444-449, 2008.

12. Levy PC, Looney RJ, Shen L, Graziano RF, Fanger MW, Roberts NJ, Ryan DH Jr and Utell MJ: Human alveolar macrophage FcR-mediated cytotoxicity. Heteroantibody- versus conventional antibody-mediated target cell lysis. J Immunol 144: 3693-3700, 1990.

13. Nishida M, Teshigawara K, Niwa O, Usuda S, Nakamura T, Ralph P, Newman R and Padlan EA: Novel humanized antiCD20 monoclonal antibodies with unique germline VH and VL gene recruitment and potent effector functions. Int $\mathrm{J}$ Oncol 32: 1263-1274, 2008.

14. Vega MI, Martinez-Paniagua, Huerta-Yepez S, GonzalezBonilla C, Uematsu N and Bonavida B: Dysregulation of the cell survival/anti-apoptotic $\mathrm{NF}-\kappa \mathrm{B}$ pathway by the novel humanized BM-ca anti-CD20 mAb: Implication in chemosensitization. Int J Oncol 35: 1289-1296, 2009.

15. Cragg MS, Morgan SM, Chan HT, Morgan BP, Filatov AV, Johnson PW, French RR and Glennie MJ: Complement mediated lysis by anti-CD20 mAb correlates with segregation into lipid rafts. Blood 101: 1045-1052, 2003.

16. Pawluczkowycz AZ, Beurskens FJ, Beum PV, Lindorfer MA, van de Winkel JG, Parren PW and Taylor RP: Binding of submaximal $\mathrm{C} 1 \mathrm{q}$ promotes complement-dependent cytotoxicity (CDC) of B cells opsonized with anti-CD20 mAbs ofatumumab (OFA) or rituximab (RTX): considerably higher levels of CDC are induced by OFA than by RTX. J Immunol 183: 749-758, 2009.

17. Cardarelli PM, Quinn M, Buckman D, Fang Y, Colcher D, King DJ, Bebbington $\mathrm{C}$ and Yarranton $\mathrm{G}$ : Binding to CD20 by anti-B1 antibody or $F\left(a^{\prime}\right) 2$ is sufficient for inhibition of apoptosis in B-cell lines. J Immunol Immunother 51: 15-24, 2002.

18. Kansas GS and Tedder TF: Transmembrane signals generated through MHC class II, CD19, CD20, CD39, and CD40 antigens induce LFA-1-dependent and independent adhesion in human B cells through a tyrosine kinase-dependent pathway. J Immunol 147: 4094-4102, 1991.

19. Sondermann P, Huber R, Oosthuizen V and Jacob U: The 3.2-A crystal structure of the human IgG1 Fc fragment-Fc gammaRIII complex. Nature 406: 267-273, 2000.

20. Shields RL, Namennuk AK, Hong K, Meng G, Rae J, Briggs J, Xie D, Lai J, Stadlen A, Li B, Fox JA and Presta L: High resolution mapping of the binding site on human IgG1 for FC $\gamma$ RI, Fc $\gamma$ RII, Fc $\gamma$ RIII, and FcRn and design of IgG1 variants with improved binding to the Fc $\gamma$ R. J Biol Chem 276: 6591-6604, 2001.

21. Gong Q, Ou Q, Ye S, Lee WP, Cornelius J, Diehl L, Lin WY, $\mathrm{Hu} \mathrm{Z}, \mathrm{Lu} \mathrm{Y}$, Chen Y, Wu Y, Meng YG, et al: Importance of cellular microenvironment and circulatory dynamics in $\mathrm{B}$ cell immunotherapy. J Immunol 174: 817-826, 2005.

22. Minard-Colin V, Xiu Y, Poe JC, Horikawa M, Magro CM, Hamaguchi Y, Haas KM and Tedder TF: Lymphoma depletion during CD20 immunotherapy in mice is mediated by macrophage Fc $\gamma$ RI, Fc $\gamma$ RII, and Fc $\gamma$ RIV. Blood 112: 1205-1213, 2008.

23. Maloney DG, Grillo-Lopez AJ, White CA, Bodkin D Schilder RJ, Neidhart JA, Janakiraman N, Foon KA, Liles TM, Dallaire BK, Wey K, Royston I, et al: IDEC-C2B8 (rituximab) anti-CD20 monoclonal antibody therapy in patients with relapsed low-grade Non-Hodgkin's lymphoma. Blood 90: 2188-2195, 1997.

24. Igarashi T, Kobayashi Y, Ogura M, Kinoshita T, Ohtsu T, Sasaki Y, Morishita Y, Murate T, Kasai M, Uike N, Taniwaki M, Kano Y, et al: Factors affecting toxicity, response and progressionfree survival in relapsed patients with indolent B-cell lymphoma and mantle cell lymphoma treated with rituximab: a Japanese phase II study. Ann Oncol 13: 928-943, 2002.

25. Jazirehi AR and Bonavida B: Cellular and molecular signal transduction pathways modulated by rituximab (rituxan, antiCD20 mAb) in non-Hodgkin's lymphoma: implications in chemosensitization and therapeutic intervention. Oncogene 24: 2121-2143, 2005.

26. Kaminski MS, Zelenetz AD, Press OW, Saleh M, Leonard J Fehrenbacher L, Lister TA, Stagg RJ, Tidmarsh GF, Krol S, Wahi RL, Knox SJ, et al: Pivotal study of iodine I 131 tositumomab for chemotherapy-refractory low-grade or transformed low-grade B-cell non-Hodgkin's lymphomas. J Clin Oncol 19: 3918-3928, 2001. 
27. Walshe CA, Beers SA, French RR, Chan CHT, Johnson PW, Packham GK, Glennie MJ and Cragg MS: Induction of cytosolic calcium flux by CD20 is dependent upon B cell antigen receptor signaling. J Biol Chem 283: 16971-16984, 2008 .

28. Nishida M, Usuda S, Okabe M, Miyakoda H, Komatsu M, Hanaoka $\mathrm{H}$, Teshigawara $\mathrm{K}$ and Niwa O: Characterization of novel murine anti-CD20 monoclonal antibodies and their comparison to 2B8 and c2B8 (rituximab). Int J Oncol 31: 29-40, 2007.

29. Maeshima AM, Taniguchi H, Nomoto J, Maruyama D, Kim SW Watanabe T, Tobinai $\mathrm{K}$ and Matsuno $\mathrm{Y}$ : Histological and immunophenotypic changes in 59 cases of B-cell non-Hodgkin's lymphoma after rituximab therapy. Cancer Sci 100: 54-61, 2008.
30. Smith MR: Rituximab (monoclonal anti-CD20 antibody): mechanism of action and resistance. Oncogene 22: 7359-7368, 2003.

31. Hiraga J, Tomita A, Sugimoto T, Shimada K, Ito M, Nakamura S, Kiyoi H, Kinoshita T and Naoe T: Down-regulation of CD20 expression in B-cell lymphoma cells after treatment with rituximab-containing combination chemotherapies: its prevalence and clinical significance. Blood 113: 4885-4893, 2009.

32. Johnson NA, Boyle M, Bashashati A, Leach S, Brooks-Wilson A, Sehn LH, Chhanabhai M, Brinkman RR, Connors JM, Weng AP and Gascoyne RD: B-cell lymphoma: reduced CD20 expression is associated with an inferior survival. Blood 113: 3773-3780, 2009. 